\title{
The Speciality Lemma, Rank 2 Bundles and Gherardelli-type Theorems for Surfaces in $\mathbf{P}^{4}$
}

\author{
MARGHERITA ROGGERO ${ }^{1 \star}$ and PAOLO VALABREGA ${ }^{2 \star}$ \\ ${ }^{1}$ Dipartimento di Matematica dell' Università, Via Carlo Alberto 10, 10123 Turin, Italy. \\ e-mail: margherita.roggero@unito.it \\ ${ }^{2}$ Dipartimento di Matematica del Politecnico, Corso Duca degli Abruzzi 24, 10129 Turin, Italy. \\ e-mail: paolo.valabrega@polito.it
}

(Received: 28 November 2001; accepted in final form 2 March 2002)

\begin{abstract}
In the present paper we give a very short and easy proof of the speciality lemma for codimension $\mathbf{2}$ subvarieties, even those that are reducible or non-reduced, in $\mathbf{P}^{n}$. Furthermore we give cohomological conditions that force a subcanonical surface in $\mathbf{P}^{4}$ to be a complete intersection and a rank 2 bundle to split, which generalize the classical First Theorem of Gherardelli.
\end{abstract}

Mathematics Subject Classifications (2000). 14F05, 14F17, 14Jxx.

Key words. Gherardelli theorems, rank 2 bundles, speciality lemma, subcanonical surfaces.

\section{Introduction and Short Account of Gherardelli-type Theorems}

The classical First Theorem of Gherardelli (see [2]) on smooth subcanonical curves in $\mathbf{P}^{3}$ can be stated as follows:

THEOREM 1 (Gherardelli). Let $X \subset \mathbf{P}^{3}$ be a smooth irreducible curve. $X$ is a complete intersection if and only if $X$ is subcanonical and the surfaces of any degree cut out complete linear series on $X$.

This theorem can be extended to arbitrary subcanonical curves, even reducible and nonreduced ones, as well as to two-codimensional subcanonical varieties lying in any projective space (see [1]). The classical proof is based on geometric arguments, while the extensions require Serre's correspondence between two-codimensional subcanonical varieties and rank 2 vector bundles in addition to cohomological techniques. In [1] the First Theorem of Gherardelli is improved as follows:

THEOREM 2 (Gherardelli-strong version). Let $X \subset \mathbf{P}^{3}$ be a locally complete intersection curve; then $X$ is a complete intersection if and only if $X$ is a-subcanonical

${ }^{\star}$ Written with the support of the University Ministry funds. 
and moreover:

- if a is even: $h^{1} \mathcal{I}_{X}\left(\frac{a}{2}+1\right)=0$;

- if a is odd: $h^{1} \mathcal{I}_{X}\left(\frac{a+1}{2}\right)=0$ or $h^{1} \mathcal{I}_{X}\left(\frac{a+3}{2}\right)=0$ or $h^{1} \mathcal{I}_{X}\left(\frac{a+5}{2}\right)=0$.

The speciality lemma and Serre's correspondence play key roles in the proof of the strong version of the theorem.

In [1] this result is extended to two-codimensional subcanonical varieties in $\mathbf{P}^{n}$. The only tool, which is straightforward, is the passage to the general hyperplane section.

The same results are also proved with cohomological techniques in [12], but only rank 2 bundles and reflexive sheaves (and no curves or varieties) are considered.

The aim of the present paper is an investigation of the strong version for subcanonical surfaces in $\mathbf{P}^{4}$ in connection with both the speciality lemma and Laudal's lemma.

Concerning the speciality lemma, we extend it to almost all two-codimensional subvarieties in $\mathbf{P}^{n}$, even reducible or nonreduced ones, provided that they are locally complete intersections, using the sheaf-based technique proposed in [10]. Such a technique allows for a very short and simple proof (as opposed to the long proof of [3]). The main tool of the proof is the following well-known theorem:

(*) for every stable rank 2 reflexive sheaf, the discriminant $c_{1}^{2}-4 c_{2}$ is stricly negative or, what amounts to the same thing for a normalized sheaf, $c_{2}>0$.

We observe that, in fact, $(*)$ and the speciality lemma are 'equivalent', i.e. the speciality lemma can be easily proved using $(*)$ and, if we assume that the speciality lemma is true, we can easily obtain $(*)$.

Theorem $(*)$, rank 2 bundles and their cohomology are key tools for our extended version of Gherardelli's theorem, while the proof in [1] for curves makes direct use of the classical form of the speciality lemma.

We also observe that Laudal's lemma has a deep connection with Gherardelli's theorem, at least in order to establish a key proposition on the vanishing of 1-cohomology groups.

In the present paper we give conditions that force a subcanonical surface in $\mathbf{P}^{4}$ to be a complete intersection and a rank 2 bundle to split. On the one hand, we simply extend Theorems 1.3, 1.6 and slightly improve Corollary 1.8 of [1]. On the other hand, we state and prove two cases that are quite new and have nothing analogous in $\mathbf{P}^{3}$. In fact in $\mathbf{P}^{3}$, the vanishing of 1 -cohomology at level $n \leqslant a / 2$ does not imply that the curve is a complete intersection, while surfaces have a different behaviour; moreover a new case arises in $\mathbf{P}^{4}$ because the null correlation bundle on $\mathbf{P}^{3}$ is not a section of a bundle on $\mathbf{P}^{4}$.

We will give two different proofs of the theorems: one is based strongly on an argument involving the hyperplane sections, while the other is based only on the characteristic function in $\mathbf{P}^{4}$ and the smallest level $\alpha$ giving a nonzero section of the bundle. Both proofs depend on a proposition on the vanishing of 1-cohomology groups, based itself on the speciality lemma and Laudal's lemma. 
Whatever proof we choose, Gherardelli's theorem for surfaces as stated here cannot be improved because of a sharp example due to Horrocks and Mumford (see Example 16).

\section{Notations and Definitions}

Unless otherwise explicitly stated:

- $\mathcal{F}$ is a rank 2 reflexive sheaf on $\mathbf{P}^{n}$ with first and second Chern classes $c_{1}$ and $c_{2}$, normalized so that $c_{1}$ is either 0 or -1 ;

$-\alpha=\alpha(\mathcal{F})$ and $\beta=\beta(\mathcal{F})(\alpha \leqslant \beta)$ are the smallest degrees of two independent generators of $H_{*}^{0} \mathcal{F}$;

- $r=r(\mathcal{F})$ is the integer $-\alpha-c_{1}$ if $\mathcal{F}$ is not stable and 0 if $\mathcal{F}$ is stable;

- the spectrum of a normalized rank 2 sheaf $\mathcal{F}$ on $\mathbf{P}^{3}$ is the unique set of $\delta=c_{2}+c_{1} r+r^{2}$ integers $\left\{k_{i}\right\}_{i=1, \ldots, \delta}$ with the following property:

$$
h^{1}(\mathcal{F}(l))=\bigoplus_{i=1}^{d} h^{0} \mathcal{O}_{\mathbf{P}^{1}}\left(k_{i}+l+1\right) \quad \text { for } l \leqslant-1
$$

it is symmetric with respect to $-\frac{c_{1}}{2}$ (see [5], Theorem 7.1 and Proposition 7.2 and [13], Theorem 3.1 and Proposition 3.5);

- $X$ is a locally Cohen-Macaulay (possibly reducible or non-reduced) codimension 2 subvariety of $\mathbf{P}^{n}$;

$-e=e(X)$ is the speciality index of $X$, that is the maximal integer $t$ such that the sheaf $\omega_{X}(-t)$ has a non-zero global section;

$-e^{\prime}=e^{\prime}(X)$ is the maximal integer $t$ such that the sheaf $\omega_{X}(-t)$ has a global section generating it almost everywhere;

- $X$ is $a$-subcanonical if the dualizing sheaf $\omega_{X}$ is isomorphic to $\mathcal{O}_{X}(a)$ for some integer $a$.

- $s=\min \left\{n / h^{0} \mathcal{I}_{X}(n) \neq 0\right\}$ is the least degree of a hypersurface containing $X$;

We will use the following well-known facts:

- if $X$ is an integral subvariety, then $e=e^{\prime}$, while in general $e^{\prime} \leqslant e$. If $X$ is the zero locus of a global section of $\mathcal{F}(t), t \gg 0$, then $e=e^{\prime}=2 t+c_{1}-n-1$, while for low values of $t$ it simply holds that $2 t+c_{1}-n-1 \leqslant e^{\prime} \leqslant e$.

- if $X$ is a subvariety in $\mathbf{P}^{n}$ and $t$ is such that $2 t+c_{1}-n-1 \leqslant e^{\prime}$ then there is a normalized reflexive sheaf $\mathcal{F}$ and a global section of $\mathcal{F}(t)$ whose zero locus is $X$; such a sheaf is a bundle if and only if $X$ is $a$-subcanonical, with $a=e^{\prime}=$ $2 t+c_{1}-n-1$ (see [5], Theorem 4.1);

- if $X$ is the zero locus of a global section of $\mathcal{F}(t)$, then

$$
s=\alpha+\beta+c_{1} \quad \text { if } t=\alpha ; \quad s=t+\alpha+c_{1} \quad \text { if } t \geqslant \beta
$$

(no codimension 2 subvariety corresponds to sections of $\mathcal{F}(t)$ if $t<\alpha$ or $\alpha<t<\beta$ ) (see [10], Lemma 1 and Remark 2); 
- if $X$ is an integral degree $d$ surface in $\mathbf{P}^{4}$, and $Y$ is its general hyperplane section, then $Y$ is an integral curve (by the second theorem of Bertini) of degree $d$ in $\mathbf{P}^{3}$; if, moreover, $X$ is $a$-subcanonical, then $Y$ is $(a+1)$-subcanonical. In this event, the arithmetic genus of $Y$ is $g=\frac{1}{2}(a+1) d+1$, which implies that if $a$ is even, $d$ must also be even.

\section{The Speciality Lemma}

In [3] Gruson and Peskine prove the following theorem concerning the smallest degree $s$ defined above in Section 2.

THEOREM 3 (Speciality lemma). Let $X \subset \mathbf{P}^{3}$ be an integral degree $d$ curve not contained in any surface of degree $<s$. Then

$$
e \leqslant s+\frac{d}{s}-4
$$

Moreover equality holds if and only if $X$ is a complete intersection of surfaces of degrees $s$ and $\frac{d}{s}$.

We will extend Theorem 3 to all dimensions as follows:

THEOREM 4. Let $X \subset \mathbf{P}^{n}$ be a two-codimensional, degree d, locally Cohen-Macaulay subvariety, generically a local complete intersection, not contained in hypersurfaces of degree $<h$ ( $h$ positive integer). Then

$$
e^{\prime} \leqslant h+\frac{d}{h}-n-1
$$

and equality holds if and only if $X$ is a complete intersection of hypersurfaces of degrees $h$ and $\frac{d}{h}$.

Following the ideas of the analogous proof given in [10] for $\mathbf{P}^{3}$, the present proof of Theorem 4 is strongly based on reflexive sheaves and their correspondence with two-codimensional varieties.

Remark 5. The above inequality can be written as follows:

$$
d \geqslant h\left(e^{\prime}+n+1-h\right)
$$

The inequality of the speciality lemma is clearly useful when written in form (1). First of all, (1) makes sense also for $h=0$, and trivially holds for every $h \leqslant 0$.

Moreover (1) has an interpretation in terms of reflexive sheaves, as the following lemma shows.

LEMMA 6. Let $\mathcal{F}$ be a rank 2 normalized reflexive sheaf and let $X$ be the subvariety corresponding to a section of $\mathcal{F}(t)$, where $t \gg 0$. If $h$ and $k$ are integers such that 
$k=h-t-c_{1}$, then the following two properties hold:

(i) $d-h\left(e^{\prime}+n+1-h\right)=c_{2}(\mathcal{F}(k))$

(ii) $h \leqslant s \Longleftrightarrow k \leqslant \alpha$.

Proof. (i) The hypotheses imply $e^{\prime}=2 t+c_{1}-n-1, d=c_{2}+t c_{1}+t^{2}$.

It is now enough to substitute in order to obtain:

$$
\begin{aligned}
d-h\left(e^{\prime}+n+1-h\right) & =c_{2}+t c_{1}+t^{2}-\left(k+t+c_{1}\right)\left(2 t+c_{1}-k-t-c_{1}\right) \\
& =c_{2}+c_{1} k+k^{2}=c_{2}(\mathcal{F}(k))
\end{aligned}
$$

Claim (ii) follows immediately if we observe that $s=t+\alpha+c_{1}$, for $t \gg 0$.

Thanks to the preceding lemma, we will see that the following theorem is in fact the speciality lemma in terms of reflexive sheaves.

THEOREM 7 (Speciality lemma for sheaves). Let $\mathcal{F}$ be a normalized reflexive sheaf. Then $c_{2}(\mathcal{F}(k)) \geqslant 0$ for all $k$ if $\mathcal{F}$ is stable, and for all $k$ outside the interval ]$\alpha,-\alpha-c_{1}[$, if $\mathcal{F}$ is nonstable.

Proof. First of all recall that $c_{2}(\mathcal{F}(k))=c_{2}+c_{1} k+k^{2}$ and $c_{2}\left(\mathcal{F}\left(-\alpha-c_{1}\right)\right)=$ $c_{2}(\mathcal{F}(\alpha)) \geqslant 0$ and equality holds if and only if the sheaf splits.

When the sheaf is stable the claim follows from the fact that the sign of $c_{2}(\mathcal{F}(k))=c_{2}+c_{1} k+k^{2}$ is always the same, because the discriminant $c_{1}^{2}-4 c_{2}$ is stricly negative (see [5], Corollary 3.3).

When the sheaf is non-stable (and hence $\alpha \leqslant-\alpha-c_{1}$ ) we have $c_{2}(\mathcal{F}(k))>$ $c_{2}(\mathcal{F}(\alpha)) \geqslant 0$ or $c_{2}(\mathcal{F}(k))>c_{2}\left(\mathcal{F}\left(-\alpha-c_{1}\right)\right) \geqslant 0$ for any integer $k$ that does not belong to the open interval $] \alpha,-\alpha-c_{1}[$.

Proof of Theorem 4. Let $\mathcal{F}$ be a normalized rank 2 reflexive sheaf such that $X$ is the zero locus of a global section of $\mathcal{F}(t)$, with $e^{\prime}=2 t+c_{1}-n-1$.

If $t \geqslant \beta$, then $s=\alpha+t+c_{1}$, so the claim is equivalent to the speciality lemma for sheaves.

If $t=\alpha$, then $s=\beta+t+c_{1}=\beta+\alpha+c_{1}$. Putting $h=\alpha+k+c_{1}$, it follows from the hypothesis that $-\alpha-c_{1}<k \leqslant \beta$. Then the claim is equivalent to $c_{2}(\mathcal{F}(k))>0$ for every $k$ belonging to the interval $\left.]-\alpha-c_{1}, \beta\right]$, and this is true because of the behaviour of the function $c_{2}(\mathcal{F}(k))=c_{2}+c_{1} k+k^{2}$.

Remark 8. As we have just seen, a proof of the speciality lemma can be based on the fact that $c_{1}^{2}-4 c_{2}$ is stricly negative, which holds for all stable rank 2 reflexive sheaves. On the other hand, if we assume that the speciality lemma holds for codimension 2 subvarieties, then it can be proved as a consequence that the discriminant $c_{1}^{2}-4 c_{2}$ is stricly negative for all stable reflexive sheaves. 
In fact we have:

$$
c_{2}(\mathcal{F}(k)) \geqslant 0 \text { for all } k \leqslant \alpha \text { if and only if } c_{2} \geqslant 0
$$

because $c_{2}(\mathcal{F}(k))=c_{2}+c_{1} k+k^{2}$ reaches its minimum value exactly when $k=0$. Observe that we can avoid the discriminant property for stable sheaves in a proof of the speciality lemma, at least in $\mathbf{P}^{3}$.

We want to point out that the discriminant property for normalized stable sheaves is equivalent to saying that $c_{2}>0$ since $c_{1}$ is either 0 or -1 .

We also wish to observe that the shortest path to the speciality lemma is based on the above mentioned property for stable sheaves, while the original proof given in [3] is long and difficult.

A useful consequence of the speciality lemma is the following corollary:

COROLLARY 9. Let $X \subset \mathbf{P}^{n}$ be a locally complete intersection two-codimensional degree $d$ subvariety. Let $p$ and $q$ be positive integers such that $p \leqslant q, p q \geqslant d$ and $p+q=e^{\prime}+n+1$.

Then either $X$ is contained in a hypersurface of degree $p-1$, or $X$ is the complete intersection of two hypersurfaces of degrees $p$ and $q=d / p$.

Proof. Let us assume that $X$ is not contained in any hypersurface of degree $p-1$. Then by Theorem 4

$$
d \geqslant p\left(e^{\prime}+n+1-p\right)=p q
$$

but $d \leqslant p q$ and hence $d=p q=p\left(e^{\prime}+n+1-p\right)$ by our assumptions. Therefore, again using Theorem $4, X$ is the complete intersection of two hypersurfaces of degrees $p$ and $q=\frac{d}{p}$.

\section{Gherardelli's Theorem for Surfaces in $\mathbf{P}^{4}$}

The aim of this section is to state and prove Gherardelli-type theorems extending Theorems 1.3 and 1.6 of [1], with some new cases.

Here $X$ will be an integral degree $d$ surface in $\mathbf{P}^{4}$ and $Y$ its general hyperplane section.

We recall the well-known Laudal lemma and some generalizations (see $[7,8,11$, 14]).

PROPOSITION 10 (Laudal's lemma). Let $X \subset \mathbf{P}^{n}$ be an integral, twocodimensional, degree $d$ variety and let $Y$ be its general hyperplane section. If the restriction map $H^{0} \mathcal{I}_{X}(t) \rightarrow H^{0} \mathcal{I}_{Y}(t)$ is not onto, then $d \leqslant t^{2}+1$.

If $n \geqslant 4$, then $d \leqslant t^{2}-t+2$ and equality holds if and only if $X$ is an arithmetically Buchsbaum surface in $\mathbf{P}^{4}$.

If $n \geqslant 5$, then $d \leqslant t^{2}-2 t+4$. 
The following proposition states a vanishing condition for 1-cohomology groups, both for an integral variety and for a reflexive sheaf. It will play a key role in the proofs of the Gherardelli-type theorems we want to present.

\section{PROPOSITION 11.}

(1) Let $X$ be an integral non-complete intersection two-codimensional subvariety in $\mathbf{P}^{n}$, $n \geqslant 4$, and let $l$ be the only integer such that either $e=2 l-n-1$ or $e=2 l-n-2$.

If $h^{1} \mathcal{I}_{X}\left(k_{0}\right)=0$ for some $k_{0} \leqslant l$, then $h^{1} \mathcal{I}_{X}(k)=0$ for all $k \leqslant k_{0}$, unless $X$ is an arithmetically Buchsbaum surface in $\mathbf{P}^{4}$ in the even liaison class of a Veronese surface.

(2) Let $\mathcal{F}$ be a non-split rank 2 reflexive sheaf on $\mathbf{P}^{n}, n \geqslant 4$.

If $h^{1} \mathcal{F}\left(t_{0}\right)=0$, for some $t_{0} \leqslant-c_{1}$, then $h^{1} \mathcal{F}(t)=0$, for every $t<t_{0}$, unless $\mathcal{F}$ is the sheaf on $\mathbf{P}^{4}$ with $c_{1}=-1$ and $c_{2}=2$ such that a general section of $\mathcal{F}(\alpha)=\mathcal{F}(2)$ corresponds to a projective Veronese surface.

Proof. First of all, observe that part (1) and part (2) of the proposition are equivalent. In our hypotheses the subvariety $X$ is the zero locus of a nonzero section of a rank 2 bundle $\mathcal{F}(l)$, where $\mathcal{F}$ is normalized and $e=2 l+c_{1}-n-1$. So $h^{1} \mathcal{I}_{X}(k)=h^{1} \mathcal{F}\left(k-l-c_{1}\right)$ for every integer $k$; moreover $k_{0} \leqslant l$ is equivalent to $t_{0}=k_{0}-l-c_{1} \leqslant-c_{1}$. So, we can only prove part (2).

The statement is true if $t_{0} \leqslant r$ : see [12] Proposition 1.7.

Now for a non-split sheaf we have $t_{0} \leqslant-c_{1} \leqslant r$ unless $t_{0}=-c_{1}=1$ and $r=0$, that is the sheaf is stable.

So it is sufficient to consider the following case:

$t_{0}=1, \quad c_{1}=-1, \quad h^{1} \mathcal{F}(1)=0 \quad$ and $\quad h^{1} \mathcal{F} \neq 0$.

Let $X$ be a subvariety corresponding to a section of $\mathcal{F}(l), l \gg 0$, and let $Y$ be its general hyperplane section; such a subvariety is integral, its degree is $d=$ $c_{2}(\mathcal{F}(l))=c_{2}-c_{1} l+l^{2}$ and the map $H^{0} \mathcal{I}_{X}(l) \rightarrow H^{0} \mathcal{I}_{Y}(l)$ is not onto.

Using Laudal's lemma (see Proposition 10) we find $d=l^{2}-l+c_{2} \leqslant l^{2}-l+2$ for $n \geqslant 4$.

But a stable sheaf on $\mathbf{P}^{3}$ (and so in $\mathbf{P}^{n}$ ) with $c_{1}=-1$ cannot have $c_{2}<2$.

Therefore the equality $d=l^{2}-l+c_{2}=l^{2}-l+2$ holds and so $n$ must be 4 . This fact can be seen in a much easier way using the last statement of Proposition 10. In any event by Laudal's lemma we find that $\mathcal{F}$ is the arithmetically Buchsbaum sheaf on $\mathbf{P}^{4}$ corresponding to the Veronese surface (see Proposition 10 or [8]).

Observe that such a sheaf is not a bundle ([9], page 113).

For vector bundles on $\mathbf{P}^{3}$ we can state a slightly different result.

LEMMA 12. Let $\mathcal{E}$ be a nonsplit rank 2 vector bundle on $\mathbf{P}^{3}$.

Then $h^{1} \mathcal{E}\left(t_{0}\right) \geqslant h^{1} \mathcal{E}\left(-t_{0}-c_{1}-4\right)$ for $-1 \leqslant t_{0} \leqslant-c_{1}$ and the equality holds if and only if $t_{0}=0$ and $\mathcal{E}$ is the null correlation bundle. 
Proof. If $\mathcal{E}$ is the null correlation bundle, then $h^{1} \mathcal{E}(t)=0$ for every $t$ except $t=-1$.

Suppose $\mathcal{E}$ is not the null correlation bundle. We want to prove that strict inequality holds.

We have

$$
\begin{aligned}
& h^{1} \mathcal{E}\left(t_{0}\right)-h^{1} \mathcal{E}\left(-t_{0}-c_{1}-4\right) \\
& \quad=\left(h^{1} \mathcal{E}\left(t_{0}\right)-h^{1} \mathcal{E}(-1)\right)+\left(h^{1} \mathcal{E}(-1)-h^{1} \mathcal{E}\left(-t_{0}-c_{1}-4\right)\right) .
\end{aligned}
$$

Using the spectrum we see that the term within the last brackets is stricly positive, while, thanks to [12] (Proposition 1.7), the term between the first brackets is nonnegative for any $t_{0} \leqslant r$ (note that $t_{0} \geqslant-1>-t_{0}-c_{1}-4$ ).

Thus, as in the proof of the previous result, it suffices to consider stable bundles with $c_{1}=-1$, in the case $t_{0}=1$.

In such a situation, using again [12] (Proposition 1.7) and the spectrum, we can see that $h^{1} \mathcal{E}-h^{1} \mathcal{E}(-1)$ is nonnegative and $h^{1} \mathcal{E}(-1)-h^{1} \mathcal{E}(-4)=\frac{3}{2} c_{2}$.

The required inequality holds if and only if $h^{1} \mathcal{E}(1)-h^{1} \mathcal{E}>-\frac{3}{2} c_{2}$.

Suppose on the contrary $h^{1} \mathcal{E}(1)-h^{1} \mathcal{E} \leqslant-\frac{3}{2} c_{2}$. Let $X$ be a curve corresponding to a global section of $\mathcal{E}(t), t \gg 0$, and let $Z$ be its general plane section. Observe that $Z$ is a set of points in uniform position (see [4]).

We then have $h^{1} \mathcal{I}_{X}(t-1)-h^{1} \mathcal{I}_{X}(t) \geqslant \frac{3}{2} c_{2} \geqslant 3$ (recall that $c_{2}$ is even and positive). By the exact sequence:

$$
h^{0} \mathcal{I}_{Z}(t) \rightarrow h^{1} \mathcal{I}_{X}(t-1) \rightarrow h^{1} \mathcal{I}_{X}(t)
$$

we can find that at least 3 curves of degree $t$ contain $Z$. The Hilbert function of $Z$ gives the upper bound $t^{2}-t+1$ for the degree of $Z$ (see [14], Section 2), which leads to a contradiction since $\operatorname{deg}(Z)=t^{2}+c_{1} t+c_{2} \geqslant t^{2}-t+2$.

As a first application of the two results given above we can extend and improve what is stated in [1]. These new results can be easily proved using the general hyperplane section and the already established Gherardelli theorem in $\mathbf{P}^{3}$.

\section{PROPOSITION 13.}

(1) Let $X \subset \mathbf{P}^{4}$ be an integral a-subcanonical surface. Then $X$ is a complete intersection if and only if $h^{1} \mathcal{I}_{X}\left(m_{0}\right)=h^{2} \mathcal{I}_{X}\left(m_{1}\right)=0$ for some integers $m_{0}, m_{1}$ such that $\frac{a}{2} \leqslant m_{1}<m_{0} \leqslant \frac{a}{2}+3$.

(2) Let $\mathcal{F}$ a normalized rank 2 vector bundle on $\mathbf{P}^{4}$. Then $\mathcal{F}$ splits if and only if $h^{1} \mathcal{F}\left(t_{0}\right)=h^{2} \mathcal{F}\left(t_{1}\right)=0$ for some integers $t_{0}, t_{1}$ such that $-2 \leqslant t_{1}<t_{0} \leqslant-c_{1}$.

Proof. Since (1) and (2) are equivalent, we prove only (2). Using Proposition 11 we can suppose $t_{0}=t_{1}+1$. If $\mathcal{F}_{H}$ is a general hyperplane section of $\mathcal{F}$, then $h^{1} \mathcal{F}_{H}\left(t_{0}\right)=0$. By Gherardelli's theorem in $\mathbf{P}^{3}$, the bundle $\mathcal{F}_{H}$ splits, and so does $\mathcal{F}$. 
The following Theorems 14 and 15 give conditions that force a subcanonical surface in $\mathbf{P}^{4}$ to be a complete intersection and a rank 2 bundle to split. We wish to point out that, while cases (a) and (b) of both theorems simply extend [1], Theorems 1.3, 1.6, and slightly improve [1], Corollary 1.8, (see also Examples 16 and 18), cases (c) and (d) are quite new and have nothing analogous in $\mathbf{P}^{3}$. We give here two different proofs of the theorems. The first proof is strongly based on an argument involving the hyperplane section, while the second proof is based only on the characteristic function in $\mathbf{P}^{4}$ and the minimal level $\alpha$ giving a nonzero section of the bundle. Of course both proofs depend upon Proposition 11 and, hence, on the speciality lemma and Laudal's lemma.

THEOREM 14. Let $X \subset \mathbf{P}^{4}$ be an integral $a$-subcanonical surface and let $b$ the only integer such that either $a=2 b$ or $a=2 b+1$.

Then $X$ is a complete intersection if and only if one of the following conditions holds:

(a) $h^{1} \mathcal{I}_{X}(b+2)=0$ and $h^{1} \mathcal{O}_{X}(b+1) \leqslant h^{1} \mathcal{O}_{X}(b+2)$

(b) $h^{1} \mathcal{I}_{X}(b+3)=0$ and $h^{1} \mathcal{O}_{X}(b+2) \leqslant h^{1} \mathcal{O}_{X}(b+3)$

(c) $h^{1} \mathcal{I}_{X}(b+1)=0, h^{1} \mathcal{O}_{X}(b) \leqslant h^{1} \mathcal{O}_{X}(b+1)$ and a is even

(d) $h^{1} \mathcal{I}_{X}(b+1)=h^{1} \mathcal{O}_{X}(b+1)=0$ and $a$ is odd.

THEOREM 15. Let $\mathcal{F}$ be a normalized rank 2 vector bundle on $\mathbf{P}^{4}$. Then $\mathcal{F}$ splits if and only if one of the following conditions holds:

(a) $h^{1} \mathcal{F}(-1)=0$ and $h^{2} \mathcal{F}(-2) \leqslant h^{2} \mathcal{F}(-1)$

(b) $h^{1} \mathcal{F}=0$ and $h^{2} \mathcal{F}(-1) \leqslant h^{2} \mathcal{F}$

(c) $h^{1} \mathcal{F}(1)=0, h^{2} \mathcal{F} \leqslant h^{2} \mathcal{F}(1)$ and $c_{1}=-1$

(d) $h^{1} \mathcal{F}(-2)=h^{2} \mathcal{F}(-2)=0$ and $c_{1}=0$

Proofs of Theorem 14 and Theorem 15 . Since they are equivalent we prove only Theorem 15. In what follows, we assume that $\mathcal{F}$ does not split and obtain a contradiction.

First proof of $(a),(b),(c)$. Let $t_{0}$ be an integer such that $-1 \leqslant t_{0} \leqslant-c_{1}$. Let $\mathcal{F}_{H}$ be a general hyperplane section of $\mathcal{F}$. Observe that the rank 2 vector bundle $\mathcal{F}_{H}$ is not the null correlation bundle, since no vector bundle $\mathcal{F}$ on $\mathbf{P}^{4}$ has $c_{2}=1$.

For $\mathcal{F}$ and $\mathcal{F}_{H}$ the following exact sequence holds:

$$
0 \rightarrow h^{1} \mathcal{F}_{H}\left(t_{0}\right) \rightarrow h^{2} \mathcal{F}\left(t_{0}-1\right) \rightarrow h^{2} \mathcal{F}\left(t_{0}\right) \rightarrow h^{2} \mathcal{F}_{H}\left(t_{0}\right) \rightarrow 0
$$

(the right-hand 0 depends upon the equality $h^{3} \mathcal{F}\left(t_{0}-1\right)=h^{1} \mathcal{F}\left(-t_{0}-c_{1}-4\right)$, whose last member vanishes since $-t_{0}-c_{1}-4<t_{0}$ and so Proposition 11 can be applied).

Thanks to that exact sequence, it is enough to prove:

$$
h^{1} \mathcal{F}_{H}\left(t_{0}\right)>h^{2} \mathcal{F}_{H}\left(t_{0}\right)=h^{1} \mathcal{F}_{H}\left(-t_{0}-c_{1}-4\right)
$$

which follows from Lemma 12. 
Second proof of $(a),(b),(c)$. Since the three cases are similar, we outline the proof of (b). when $c_{1}=0$.

We recall that $c_{2}+\alpha^{2}>0$ if and only if $\mathcal{F}$ is non-split.

Put $\Delta=h^{2} \mathcal{F}-h^{2} \mathcal{F}(-1)$ and consider the difference $\chi(\mathcal{F})-\chi(\mathcal{F}(-1))$ of the Euler characteristic functions: $\chi(\mathcal{F})-\chi(\mathcal{F}(-1))=-2 c_{2}$. If $\alpha<0$, then we have $\Delta=$ $-2\left(c_{2}+\alpha^{2}\right)<0$ (because $c_{2}+\alpha^{2}$ is the degree of the minimal surface of the bundle). If $\alpha=0$, the $\Delta=1-2\left(c_{2}+\alpha^{2}\right) \leqslant-1$. If $\alpha>0$, the $\Delta=2-2 c_{2}<0$.

Proof of $(d)$. The hypothesis implies that $h^{1} \mathcal{F}_{H}(-2)=0$; so the spectrum of $\mathcal{F}_{H}$ contains only 0 . This is not allowed if $\mathcal{F}_{H}$ is nonstable (see [13], Proposition 3.2).

So assume that $\mathcal{F}_{H}$ is semistable. Then we have: the Euler characteristic function $\chi(\mathcal{F}(-2))=\frac{1}{12}\left(c_{2}+1\right) c_{2}$. This implies either $c_{2}=0$ or $c_{2}=-1$, both of which are forbidden values for a semistable bundle.

EXAMPLE 16. Let $\mathcal{F}$ be the normalized Horroks-Mumford non-split vector bundle on $\mathbf{P}^{4}$ whose $c_{1}$ is -1 (see [6]). We have: $h^{2} \mathcal{F}(t)=0$ for every $t \neq-2$ and $h^{1} \mathcal{F}(t)=0$ for every $t \leqslant-1$. So the first half of condition (a) holds, while the second half does not, and the second half of both conditions (b). and (c). holds, while the first half does not.

Remark 17. In [1] a result analogous to (a), (b), (c) is proved. Instead of the inequality $h^{2} \mathcal{F}(t) \leqslant h^{2} \mathcal{F}(t+1)$, in [1] the vanishing of $h^{2} \mathcal{F}(t)$ is assumed. The following example shows that the condition in the present paper is in fact weaker. We observe also that the conditions of the theorem do not force a reflexive sheaf to split, they force only a bundle to split, as is shown in Example 19.

EXAMPLE 18. Let $M$ be a Buchsbaum graded $k\left[X_{0}, \ldots, X_{4}\right]$-module with homogeneus components $M_{0}=M_{1}=k$ and $M_{i}=0$ for all $i \neq 0,1$. Then there exists a rank 2 reflexive sheaf $\mathcal{F}$ on $\mathbf{P}^{4}$ (respectively: an integral Cohen-Macaulay surface in $\left.\mathbf{P}^{4}\right)$ such that its 1-cohomology vanishes and its module of 2-cohomology is $M(-t)$, for some $t$. Then $h^{2} \mathcal{F}(t) \leqslant h^{2} \mathcal{F}(t+1)$, but $h^{2} \mathcal{F}(t) \neq 0$.

EXAMPLE 19. Let $X$ be a 2-plane in $\mathbf{P}^{4}$ and let $\mathcal{F}$ be the reflexive sheaf corresponding to $X$ through a section of $\omega_{X}(-4)$. Then the 1 and 2-cohomology vanish everywhere but $\mathcal{F}$ is not a split bundle.

\section{References}

1. Chiantini, L. and Valabrega, P.: Subcanonical curves and complete intersections in projective 3-space, Ann. Mat. Pura Appl. 138(4) (1984), 309-330.

2. Gherardelli, G.: Sulle curve sghembe algebriche intersezioni complete di due superficie, Atti Reale Accad. Ital. 4 (1943), 128-132.

3. Gruson, L. and Peskine, C.: Genre des courbes de l'espace projectif, In: Algebraic Geometry (Proc. Sympos., Univ. Tromsø, Tromsø, 1977), Lecture Notes in Math 687, Springer, Berlin, 1978, pp. 31-59.

4. Harris, J.: The genus of space curves, Math. Ann. 249 (1980), 191-204. 
5. Hartshorne, R.: Stable reflexive sheaves, Math. Ann. 254 (1980), 121-176.

6. Horrocks, G. and Mumford, D.: A rank 2 vector bundle on $P^{4}$ with 15,000 symmetries, Topology 12 (1973), 63-81.

7. Laudal, O.: A generalized trisecant lemma, In: Algebraic Geometry Proceedings (Tromsø 1977), Lecture Notes in Math., 687, Springer, Berlin, 1978, pp. 112-149.

8. Mezzetti, E.: The border cases of the lifting theorem for surfaces in $P^{4}$, J. Reine Angew. Math. (1992), 101-111.

9. Okonek, C., Schneider, M. and Spindler, H.: Vector bundles on complex projective spaces, Progr. in Math. 3 (1990).

10. Roggero, M.: Some remarks about the speciality theorem of Gruson and Peskine, Atti Acc. Sci. Torino 119, V-VI, (1985), 253-256.

11. Roggero, M.: Laudal-type theorems in $P^{n}$, To appear on: Indag. Math.

12. Roggero, M. and Valabrega, P.: Some vanishing properties of the intermediate cohomology of a reflexive sheaf on $P^{n}, J$. Algebra 170(1) (1994), 307-321.

13. Sauer, T.: Nonstable reflexive sheaves on $P^{3}$, Trans. Amer. Math. Soc. 281 (1984), 633-655.

14. Valenzano, M.: Bounds on the degree of two-codimensional integral varieties in projective space, J. Pure Appl. Algebra 158 (2001), 111-122. 